\title{
Assessing a cognitive rehabilitation environment based on interactive video and eye-tracking technologies
}

\author{
Martínez-Moreno JM, Solana J, \\ Sánchez-González P, Gómez EJ
}

\author{
Sánchez-Carrión R, López J, \\ Roig T, Tormos JM
}

\begin{abstract}
This paper presents an study design to assess the effectiveness of a new cognitive rehabilitation environment based on interactive video and advanced monitoring technologies.

Keywords-Neurorehabilitation; cognitive rehabilitation; virtual environments; interactive video; eye-tracking; evaluation study
\end{abstract}

\section{INTRODUCTION}

The use of new technologies in neurorehabilitation has led to higher intensity rehabilitation processes, extending therapies in an economically sustainable way. Interactive Video (IV) technology allows therapists to work with virtual environments that reproduce real situations. In this way, patients deal with Activities of the Daily Living (ADL) immersed within enhanced environments [1]. These rehabilitation exercises, which focus in re-learning lost functions, try to modulate the neural plasticity processes [2].

A cognitive rehabilitation environment based on the IV and eye-tracking (ET) technologies integration is proposed. This work presents an study design to assess the effectiveness of these technologies in order to support therapeutic interventions as part of the cognitive rehabilitation therapy.

IV and ET may be effective technologies to be used in the cognitive rehabilitation therapies attending to these both hypotheses:

- The proposed environment based on IV and ET allows the detection of abnormal cognitive behavior.

- The design of therapeutic intervention strategies is supported by the IV-based ADL, helping subjects to achieve the ADL's goal more efficiently: focusing user attention to the important stimuli, and both reducing the execution time and the number of errors.

\section{COGNITIVE REHABILITATION ENVIRONMENT}

\section{A. Interactive Video}

IV refers to any video whose sequences and displayed information depend on the user's responses [3]. Interactivity is provided by associating an interaction with any element which appears in the video scenes. The video flow is modified according to the way users interact with these elements.

Several researchers highlighted the IV technology capacity for adapting the learning process to each user's capacities $[4,5]$, and it may indeed accelerate skill acquisition [6]. Moreover, some studies proved that ADL observation treatments are a good rehabilitation approach in stroke patients [7]. Preziosa et al. evidenced that even observing activity in videos could remodel the brain due to mirror neuron system activation [8].

In this context, IV is proposed as a technology for developing virtual environments where ADLs are reproduced [3]. These environments are based on real images and easy navigation with simple and controlled user's interactions. Therefore, IV-based ADLs offer neuropsychologists the opportunity to plan therapeutic intervention strategies depending on the cognitive capacities of each patient.

\section{B. Eye-tracking}

ET technology is proposed to support and assess user's activity performance. ET provides information about user visual attention which is closely related with his/her cognitive state [9].

The ET device Tobii 1750 [10] is integrated with the IVbased cognitive rehabilitation environment [11]. Thus, the system captures visual attention data while the user is performing the activity.

Knowledge on user's attention focus, his/her cognitive state and the validity of the rehabilitation task can be extracted from gaze data [12]. Moreover, matching this information with the user's activity performance data, provides knowledge on the attentional circumstances in which he/she took each decision. 
This information is used to properly adapt the activity and the therapeutic interventions to the user's cognitive capacities.

\section{ADL "Buying Bread"}

Neuropsychologists from Instituto Guttmann designed the ADL "Buying Bread" [3]. The ADL reproduces the steps that a subject, who is initially sitting on the sofa, has to follow in order to go to the street, find the bakery and buy bread. The ADL is divided in three tasks corresponding to the three different stages where the activity is performed: "home", "street" and "bakery". Task's goals were "picking up personal belongings and leaving home", "going to the bakery", and "buying bread", respectively.

The three tasks were designed according to a successive approximation model [13] where patients must achieve shortterms goals interacting with the environment in order to achieve task's goal. Neuropsychologists designed a therapeutic interaction protocol which is applied to every single interaction and based on a three assistance levels (the aid intensity depends on the patient's needs). Thus, the patient is guided along the activity according to an errorless learning [14].

The ADL "Buying Bread" was developed using IV technology and integrated with the ET system.

\section{EVALUATION STUDY}

\section{A. Objectives}

An empirical study is being performed in order to evaluate the stated hypotheses. The main goal is to assess IV and ET as development technologies of cognitive rehabilitation environments to support therapeutic interventions based on ADLs.

For this purpose, this study establishes the evaluation of: (a) the effectiveness of the therapeutic intervention upon the patient's ADL performance; (b) the environment's capacity to monitor and detect abnormal behaviors depending on users' cognitive capacities; and (c) the usability of the IV-based cognitive rehabilitation environment.

\section{B. Design and Methodology}

Two groups of people participate in the study: a group of healthy people, and a group of patients with a cognitive deficit. At the same time, each group is divided into two subgroups depending on the version of ADL they perform: (a) Guided ADL, according to the therapeutic intervention design; or (b) Non Guided ADL, only one instruction is given at the beginning of the ADL. Each subgroup is comprised of 20 subjects, aiming for gender balance.

Every single subject performs once the ADL "Buying Bread" monitored by the eye-tracker. Data log will be extracted from each performance, attending to three different points of view:

- Usability evaluation according to the user experience.

- Activity data: execution time, number of hits/errors and perseverance.
- Gaze data: time-stamped data on the screen coordinates, attention deficits, fixations in every stimulus and heat maps.

\section{DISCUSSION}

Stated hypothesis about the effectiveness of the proposed cognitive rehabilitation environment will be evaluated. If confirmed, new ADLs would be designed and developed using IV in order to become integrated into routine clinical practice.

New means of interaction are being taken into a consideration with the aim of making the user experience as accessible as possible. Touch screens or gesture devices could be integrated with the proposed cognitive rehabilitation environment.

\section{ACKNOWLEDGMENT}

The authors thank Samuel González from Lavinia Interactiva for its collaboration during the development of the environment. This research has been partially founded by the Spanish Ministry of Economy and Finance (project TIN201238450, COGNITIO).

\section{REFERENCES}

[1] Gamito P, Oliveira J, Morais D, Rosa P, Saraiva T. NeuAR-A Review of the VR/AR Applications in the Neuroscience Domain. In: Dr. Andrew Yeh Ching Nee, editor. Augmented Reality - Some Emerging Application Areas: InTech; 2011

[2] Pascual-Leone A, Amedi A, Fregni F, Merabet L. The plastic human brain cortex. Annu Rev Neurosci 2005;28:377-401.

[3] Martinez-Moreno JM, et al. Cognitive Neurorehabilitation based on Interactive Video Technology. Informatics, Management and Technology in Healthcare2013;190:27-29.

[4] Zhang D, Zhou L, Briggs RO, Nunamaker Jr JF. Instructional video in elearning: Assessing the impact of interactive video on learning effectiveness. Information \& Management 2006;43(1):15-27.

[5] Merkt M, Weigand S, Heier A, Schwan S. Learning with videos vs. learning with print: The role of interactive features. Learning and Instruction 2011;21(6):687-704.

[6] Schwan S, Riempp R. The cognitive benefits of interactive videos: Learning to tie nautical knots. Leaming and Instruction 2004;14(3):293 305 .

[7] Franceschini M, et al. Mirror neurons: action observation treatment as a tool in stroke rehabilitation. Eur J Phys Rehabil Med 2010;46(4):517523 .

[8] Preziosa P, et al. Action observation therapy modifies structural brain plasticity in healthy adult individuals. Eur J Neurol 2014;21:79-79.

[9] Suh M, et al. Degree of brain connectivity predicts eye-tracking variability. Journal of Korean Physical Society 2008;53:3468-3473.

[10] Tobii Technology. Tobii. 2014; Available at: http://www.tobii.com/. Accessed Enero, 2015.

[11] Martinez Moreno JM, et al. Monitoring visual attention on a neurorehabilitation environment based on Interactive Video. Proceedings of International Conference on Recent Advances in Neurorehabilitation. Valencia. 2013.

[12] Duchowski A. Eye tracking methodology: Theory and practice. Springer; 2007.

[13] Wilson BA, Herbert CM, Shiel A. Behavioural approaches in neuropsychological rehabilitation: Optimising rehabilitation procedures. Psychology Press; 2003.

[14] Instructional techniques in cognitive rehabilitation: A preliminary report. Seminars in speech and language: Theime Medical Publishers; 2005. 